\title{
Benign Presentation Following Massive Deutetrabenazine Overdose
}

\author{
Oluseyi Obadeyi ${ }^{1}$, James H. Paxton ${ }^{2}$, Sarkis Kouyoumjian ${ }^{2}$ \\ 1. Emergency Medicine, Wayne State University School of Medicine, Detroit, USA 2. Emergency Medicine, Wayne State \\ University Detroit Medical Center, Detroit, USA
}

Corresponding author: Oluseyi Obadeyi, sobadeyi@gmail.com

\begin{abstract}
Deutetrabenazine (DTBZ) (Austedo ${ }^{\circledR}$ ) is a novel vesicular monoamine transporter 2 (VMAT2) inhibitor, which acts by blocking dopamine release and other monoamines from neuronal vesicles. Although this drug is considered the first-line treatment for tardive dyskinesia (TD), VMAT2 inhibition has also been shown to improve patients' symptoms with Huntington's disease-induced chorea. We present the case of a 59-yearold woman with a history of TD, who presented to the emergency department following massive DTBZ ingestion. The relative paucity of other overdose symptoms further supports the manufacturer's claims of a low side effect profile for this drug in overdose. Although DTBZ demonstrates an excellent safety profile, emergency physicians should be aware of the potential side effect of DTBZ overdose, in addition to other known side effects of this novel drug.
\end{abstract}

Categories: Emergency Medicine, Neurology, Other

Keywords: deutetrabenazine, overdose, toxicology, side effects, toxicity, tardive dyskinesia

\section{Introduction}

Deutetrabenazine (DTBZ) (Austedo ${ }^{\circledR}$ ) is a novel vesicular monoamine transporter 2 (VMAT2) inhibitor that blocks dopamine release and other monoamines from neuronal vesicles. Although this drug is considered the first-line treatment for tardive dyskinesia (TD), VMAT2 inhibition has been shown to improve patients' symptoms with Huntington's disease (HD)-induced chorea [1]. TDs are involuntary movements of the tongue, lips, face, trunk, or extremities associated with and often attributed to long-term use of dopaminergic antagonist medications [1]. The unique movements of TD are repetitive, purposeless, and involuntary and can often cause functional impairment. We present the case of a 59-year-old woman with a history of TD, who presented to the Emergency Department (ED) following massive DTBZ ingestion. The patient had an benign course.

Review began $12 / 26 / 2020$ Review ended 01/21/2021 Published 01/24/2021

\section{Copyright 2021}

Obadeyi et al. This is an open access article distributed under the terms of the Creative Commons Attribution License CC-BY 4.0., which permits unrestricted use, distribution, and reproduction in any medium, provided the original author and source are credited.

\section{Case Presentation}

A 59-year-old female with a history of hypertension, type 2 diabetes, hypothyroidism, TD, Parkinson's disease, schizophrenia, bipolar disorder, and ischemic stroke presented to the ED with her daughter for DTBZ overdose. Her daughter stated that the patient ingested 20 tablets of $12 \mathrm{mg}$ DTBZ (Table 1) approximately more than eight hours before presenting to the ED. She denied wanting to hurt herself and having any history of a suicide attempt. Our patient stated that she took the medication because she thought it was a pain medication that would relieve a left lower extremity pain that she sustained from hitting her left lower leg on a bed frame. She admitted to feeling depressed, and her only complaint was that she felt drowsy. She had no other symptoms or complaints. The patient denied headache, tinnitus, nausea, vomiting, chest pain, shortness of breath, or any other symptoms.

Her vital signs on arrival revealed a temperature of $98.8^{\circ} \mathrm{F}$, blood pressure of $162 / 104 \mathrm{mmHg}$, heart rate of 74 beats per minute, respiration of 20 breaths per minute, and oxygen saturation $\left(\mathrm{SpO}_{2}\right)$ of $96 \%$ on room air. Physical examination revealed an alert and oriented female who was in no acute distress. She had clear bilateral lung sounds, non-labored respirations with a regular heart rate, and rhythm. Aside from tremors noted on her right hand, she had unremarkable sensory, strength, and deep tendon reflexes in all extremities. 


\section{Cureus}

\begin{tabular}{|l|l|}
\hline Drug & Dose \\
\hline Aspirin & $81 \mathrm{mg}$ by mouth, daily \\
\hline Atorvastatin & $40 \mathrm{mg}$ by mouth, daily \\
Carvedilol & $12.5 \mathrm{mg}$ by mouth, twice daily \\
Clopidogrel & $75 \mathrm{mg}$ by mouth, daily \\
\hline Deutetrabenazine & $12 \mathrm{mg}$ by mouth, daily \\
\hline Docusate & One Cap $100 \mathrm{mg}$ by mouth, twice daily \\
\hline Hydralazine & $75 \mathrm{mg}$ by mouth, three times daily \\
\hline Levothyroxine & $125 \mathrm{mCg}$ by mouth, daily \\
Nifedipine & Tab $90 \mathrm{mg}$ by mouth, daily \\
\hline Quetiapine & Tab $50 \mathrm{mg}$ by mouth, before bed \\
\hline
\end{tabular}

TABLE 1: Patient's outpatient medications prior to overdose presentation

Mg: milligrams, mCg: microgram, Cap: capsule

Electrocardiogram demonstrated normal sinus rhythm. She had a narrowed anion gap, and her complete blood count and electrolyte levels were within normal limits. Her urine drug screen for amphetamines, barbiturates, benzodiazepines, cannabinoids, cocaine metabolite, methadone, and opiates was negative.

The toxicology service was consulted and recommended 23 hours of telemetry observation to monitor for QT prolongation. The patient had an uneventful overnight course. The next morning, her orofacial TD was present, and neurology was consulted. Neurology recommended psychiatry consult to evaluate for suicidal ideation and outpatient follow-up to discuss the resumption of DTBZ. From a medical standpoint, the patient had an unremarkable hospital course. She was medically cleared by the neurology and inpatient team on day two of her admission. She was admitted for three days, and her length of stay was prolonged because she reported to the psychiatry team that she would like to be placed in a group home facility instead of living with her daughter at home. The patient was finally discharged home with her daughter after changing her mind about desiring a group home placement.

\section{Discussion}

Before the introduction of VMAT2 inhibitors, TD was believed to be an irreversible side effect of dopamine antagonism. Neuroleptics and antiemetic dopamine blocking agents cause TD as a side effect of their clinical use. First- and second-generation antipsychotic treatments have been linked with a 5.5\% and 3.9\% yearly incidence rate of TD development, respectively [1]. Although the pathophysiology of TD development is poorly understood, it is thought to involve the upregulation and sensitization of the dopamine D2 receptor as a result of the prolonged blockade [2].

DTBZ functions as a reversible VMAT2 inhibitor. The US Drug Administration (FDA) approved it in August 2017 for the treatment of TD in adults. The medication is also approved to treat HD-induced chorea and Tourette syndrome [3-5]. Tetrabenazine (TBZ) is the archetype VMAT2 inhibitor and was used for decades to manage hyperkinetic movement disorders before developing other VMAT2 inhibitors like DTBZ and valbenazine (Ingrezza ${ }^{\circledR}$ ). The substandard pharmacokinetic profile and lack of FDA approval other than its use for HD chorea limited the use of TBZ in the US. Valbenazine and DTBZ addressed some of the deleterious pharmacokinetic profile of TBZ. Although TBZ and DTBZ are structurally similar, the substitution of deuterium for hydrogen in strategic locations of TBZ resulted in a compound with better pharmacokinetics and safety profile [6-8]. At approximately half the dose of TBZ, DTBZ offers a comparable total exposure time with a longer half-life and at a lower maximum serum concentration [8].

Although it is safer than TBZ, DTBZ still has the potential to cause serious side effects. A 12-week trial examining patients with HD revealed that somnolence was the most common dose-limiting side effect of DTBZ [9]. The other adverse effects noted in TD patients include nasopharyngitis, insomnia, depression, and akathisia [3,9]. DTBZ can induce QT prolongation in patients who are CYP2D6 poor metabolizers or consuming a potent CYP2D6 inhibitor. DTBZ also has the potential to cause hyperprolactinemia and neuroleptic malignant syndrome (NMS) [3]. Patients with NMS present with fever, muscle rigidity, and altered mental status with autonomic changes. The novelty of DTBZ demonstrates the lack of sufficient 
In this case, the patient had no significant adverse effect from a substantial DTBZ consumption at the time of initial presentation. DTBZ for the management of TD appears to be well-tolerated. The recommended starting dose of DTBZ for the management of TD is $6 \mathrm{mg} /$ day, and it can be titrated up to a recommended maximum dose of $48 \mathrm{mg}$ per day. Previous studies started DTBZ at $12 \mathrm{mg}$ /day (6 mg twice daily) with weekly titration of $6 \mathrm{mg} /$ day for up to six weeks until the maximum $48 \mathrm{mg}$ per day or adequate TD control was reached, barring the occurrence of a severe side effect [7]. Due to the ability of TBZ to cause QT interval prolongation, it is recommended that patients at risk of developing QT prolongation have their QT interval assessed before and after increasing their total dose above $24 \mathrm{mg}$ per day [3].

The diagnosis of DTBZ overdose can only be made through history taking and clinical presentation. Management of DTBZ overdose includes discontinuation of DTBZ, symptomatic treatment, and medical monitoring. DTBZ metabolites are primarily metabolized by CYP2D6 and virtually eliminated renally [3]. Although she had ingested ten times her prescribed daily dose of DTBZ, the patient described in this case report had a mild symptom. The only symptom that she complained of was somnolence, which is the most common adverse effect of DTBZ in patients [3,7,9]. The reported half-life of the active metabolites of DTBZ is nine to ten hours [3]. Considering the substantial amount of DTBZ the patient ingested, a noteworthy observation was that the patient's orofacial TD manifested the following morning after her admission 24 hours after DTBZ ingestion.

\section{Conclusions}

DTBZ overall has an excellent safety profile with tolerable side effects when utilized for the management of TD. Because of its novelty, limited studies have investigated the potential clinical presentation of DTBZ poisoning. Our patient had a mild symptom following massive DTBZ ingestion. Although DTBZ demonstrates an excellent safety profile, emergency physicians should be conscious of its potential to cause fetal abnormalities like QT prolongation and NMS. Consultation with a medical toxicologist and heart monitoring for QT prolongation is recommended.

\section{Additional Information}

\section{Disclosures}

Human subjects: Consent was obtained or waived by all participants in this study. Conflicts of interest: In compliance with the ICMJE uniform disclosure form, all authors declare the following: Payment/services info: All authors have declared that no financial support was received from any organization for the submitted work. Financial relationships: All authors have declared that they have no financial relationships at present or within the previous three years with any organizations that might have an interest in the submitted work. Other relationships: All authors have declared that there are no other relationships or activities that could appear to have influenced the submitted work.

\section{References}

1. Carbon M, Hsieh C-H, Kane JM, Correll CU: Tardive dyskinesia prevalence in the period of secondgeneration antipsychotic use: a meta-analysis. J Clin Psychiatry. 2017, 78:264-78. 10.4088/JCP.16r10832

2. Waln O, Jankovic J: An update on tardive dyskinesia: from phenomenology to treatment . Tremor Other Hyperkinet Mov. 2013, 3:1-11. 10.7916/d8bg304w

3. Teva Pharmaceuticals: AUSTEDO (deutetrabenazine) tablets, for oral use. Prescribing information. North Wales, PA; 2017.

4. Neurocrine Biosciences: INGREZZA (valbenazine) capsules, for oral use. Prescribing information. San Diego, CA; 2017.

5. Jankovic J, Jimenez-Shahed J, Budman C, Coffey B, Murphy T, Shprecher D, Stamler D: Deutetrabenazine in tics associated with Tourette syndrome. Tremor Other Hyperkinet Mov. 2016, 6:422. 10.7916/D8M32W3H

6. Jankovic J: Dopamine depleters in the treatment of hyperkinetic movement disorders . Expert Opin Pharmacother. 2016, 17:2461-70. 10.1080/14656566.2016.1258063

7. Fernandez HH, Factor SA, Hauser RA, et al.: Randomized controlled trial of deutetrabenazine for tardive dyskinesia: the ARM-TD study. Neurology. 2017, 88:2003-10. 10.1212/WNL.0000000000003960

8. Stamler D, Brown F, Bradbury M: The pharmacokinetics of extended release SD-809, a deuteriumsubstituted analogue of tetrabenazine. Mov Disord. 2013, 28:765.

9. Frank S, Testa CM, Stamler D, et al.: Effect of deutetrabenazine on chorea among patients with Huntington Disease: a randomized clinical trial. JAMA. 2016, 316:40-50. 10.1001/jama.2016.8655 